\title{
Silencing of osterix expression by siRNA inhibits aldosterone-induced calcification of vascular smooth muscle cells in mice
}

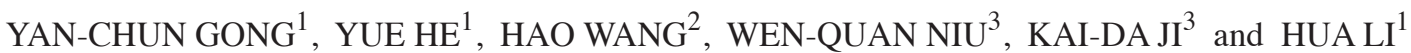 \\ ${ }^{1}$ Department of Hypertension, Shanghai Institute of Hypertension, Ruijin Hospital, Shanghai Jiao Tong University \\ School of Medicine, Shanghai 200025; ${ }^{2}$ The Experimental Center of Basic Medicine, Shanghai Medical College, \\ Fudan University, Shanghai 200032; ${ }^{3}$ Shanghai Key Laboratory of Vascular Biology, Shanghai Institute of Hypertension, \\ Ruijin Hospital, Shanghai Jiao Tong University School of Medicine, Shanghai 200025, P.R. China
}

Received May 28, 2015; Accepted June 10, 2016

DOI: $10.3892 / \mathrm{mmr} .2016 .5484$

\begin{abstract}
The process of vascular calcification shares numerous similarities with that of skeletal mineralization and involves the deposition of hydroxyapatite crystals in arteries and cardiac valves. However, the underlying cellular mechanism remains to be fully elucidated. Microarray analysis in the present study demonstrated that greater than 2,000 genes were upregulated during the calcification of murine vascular smooth muscle cells (VSMCs), of which osterix (OSX) and integrin-binding sialoprotein (IBSP) were the most significantly differentially expressed genes. Following the validation of increased OSX and IBSP expression by reverse transcription-quantitative polymerase chain reaction in calcifying murine VSMCs induced by aldosterone. Subsequent to transfection with siRNA-OSX, results indicated that OSX may inhibit calcification of VSMCs via IBSP. It was suggested that the increased OSX expression in calcifying VSMCs may reflect the well-established prenatal role of OSX. A full understanding of the importance of OSX in this pathological process would improve understanding of the pathogenesis of vascular calcification.
\end{abstract}

\section{Introduction}

Osterix (OSX) is predominantly expressed in osteoblasts and cells associated with tooth development during the human fetal stage, and in craniofacial osteoblasts and chondrocytes after birth, although there is additionally low-level expression

Correspondence to: Dr Yan-Chun Gong, Department of Hypertension, Shanghai Institute of Hypertension, Ruijin Hospital, Shanghai Jiao Tong University School of Medicine, 197 Ruijin 2nd Road, Shanghai 200025, P.R. China

E-mail: yanchungong@126.com

Key words: vascular smooth muscle cell, calcification, siRNA, aldosterone, osterix in the testis, heart, brain, placenta, lung, pancreas, spleen and myofibroblasts of major arteries. OSX is also highly expressed in certain tumor cells including those of osteosarcoma cells and giant cell tumor mesenchymal cells, and is additionally associated with tumor biological behaviors (1-3).

OSX is an osteoblast-specific transcription factor with a zinc ion motif structure domain that is required in the process of osteocyte differentiation and bone formation. It is located downstream of the runt-related transcription factor- 2 gene as a specific transcription factor that controls osteoblast growth and differentiation $(4,5)$, which is specifically expressed in developed bone tissues (6). Without OSX participation, bone formation within the membrane or cartilage becomes impossible (4), and therefore OSX is the key transcription factor in osteoblast differentiation.

Vascular calcification is a pathological process universally present in atherosclerosis, hypertension and renal vasculopathies. The dualistic function of calcified vessels is reduced and stiffness will be increased, thus facilitating the development of thrombosis and rupture of atherosclotic plaques. Approximately $80 \%$ patients with vascular injuries and $90 \%$ of patients with coronary artery disease experience vascular calcification (7). A previous study (8) demonstrated that vascular calcification is a cell-controlled and highly regulated process similar to the active and regulatory process of bone growth and osteoporosis. Aldosterone (ALD) is the main component of adrenal mineralocorticoid hormone. ALD results in sodium and water retention, in addition to promoting collagen deposition and fibrosis, leading to structural remodeling of the heart and blood vessels, thus serving an important role in the development and progression of vascular calcification (9).

The elucidation of a postnatal role of OSX in the survival of progenitor pools in their interactions with the stem cell niches (10), together with evidence associating OSX with vascular function (11), has led to the hypothesis that OSX may be a key mediator of postnatal phenotypic trans-differentiation of vascular smooth muscle cells (VSMCs) during calcification. To the best of our knowledge, this is the first study to investigate the role of OSX in the process of vascular calcification. The current in vitro study investigating VSMC 
calcification aimed to provide the first fundamental insight into the expression profiles of OSX during vascular calcification.

In the present study, ALD was used to induce calcium salt deposition in cultured mouse VSMCs, the gene chip technique was used to analyze differentially expressed genes of calcified VSMCs and OSX-specific VSMC small interfering RNA (siRNA) was used to silence the OSX gene in order to verify the effect of downregulation of OSX on the calcification of VSMCs.

\section{Materials and methods}

Isolation of primary murine VSMCs. Primary VSMCs were isolated from 6-week-old male C57BL/6 mice ( $\mathrm{n}=6 ; 20-30 \mathrm{~g}$ ). All animals were housed in Makrolon cages, and provided an extruded diet and acidified tap water ad libitum. Lighting was maintained on a 12:12 dark/artificial light cycle with lights on from $6.00 \mathrm{a} . \mathrm{m}$. and with a 30 -min period of reduced lighting at transitions. The temperature was maintained at $22 \pm 2^{\circ} \mathrm{C}$ ) with a relative humidity of $55 \%$. The mice were sacrificed by $\mathrm{CO}_{2}$ inhalation. Following dissection of the aorta, the adventitia was detached and the blood vessel was opened to expose the endothelial layer of cells. The aortae isolated from 16 mice were then digested with $1 \mathrm{mg} / \mathrm{ml}$ trypsin for $10 \mathrm{~min}$ to remove any remaining endothelium and adventitia. This was followed by an overnight incubation at $37^{\circ} \mathrm{C}$ in a humidified atmosphere of $95 \%$ air $/ 5 \% \mathrm{CO}_{2}$ in $\alpha$-minimum essential medium ( $\alpha$-MEM; GE Healthcare Life Sciences, Logan, UT, USA) supplemented with $10 \%$ fetal calf serum (GE Healthcare Life Sciences) and $1 \%$ gentamycin (GE Healthcare Life Sciences). Tissues were then digested in $425 \mathrm{U} / \mathrm{ml}$ collagenase type II for $5 \mathrm{~h}$. Prior to the experiment, the isolated VSMCs were expanded in $\alpha$-MEM for two passages in T25 tissue culture flasks (Greiner Bio-One GmbH, Frickenhausen, Germany) coated with $0.28 \mathrm{mg} / \mathrm{cm}^{2}$ murine laminin (Sigma-Aldrich, St. Louis, MO, USA). The current study was approved by the ethics committee of Shanghai Medical College, Fudan University (Shanghai, China).

Culture of primary murine VSMCs. VSMCs were plated at $1.5 \times 10^{4} / \mathrm{cm}^{2}$ in a 6 -well tissue culture plate containing $\alpha$-MEM. To induce calcification of VSMCs, $1.5 \mathrm{mmol} / \mathrm{l}$ ALD (Sigma-Aldrich) was added to confluent cells for 6 days, and cells were then incubated at $37^{\circ} \mathrm{C}$ in a humidified atmosphere of $95 \%$ air $/ 5 \% \mathrm{CO}_{2}$. The medium was replaced every second day.

cDNA microarray hybridization. RNA was extracted from cells using the RNeasy Mini kit (Qiagen GmbH, Hilden, Germany) according to the manufacturer's instructions for microarray analysis. Total RNA content was assessed by absorbance at $260 \mathrm{~nm}$ and purity by A260/A280 ratio for each sample. The quality of each sample was considered suitable if it had a ratio $>1.9$. A $1 \mu \mathrm{g}$-labeled probe was hybridized at $65^{\circ} \mathrm{C}$ for the 17 -h rolling hybridization. The microarray result was scanned using an Agilent Microarray Scanner (Agilent Technologies, Inc., Santa Clara, CA, USA), and the data were read with Feature Extraction software, version 10.7 (Agilent Technologies, Inc.) and analyzed using the standard method.
Microarray data analysis. Analysis was conducted as described previously (12). Normalization across all arrays was achieved using the robust multi-array average expression measure which results in expression measures (summarized intensities) in log base 2 (13). Comparisons were performed using linear modeling. Following this, empirical Bayesian analysis was conducted including vertical (within a given comparison) P-value adjustments for multiple testing, which control for false discovery rates using the Bioconductor package limma (14).

Detection of calcification. Calcium deposition was evaluated through decalcification of the mineralized matrix in $0.6 \mathrm{M} \mathrm{HCl}$ for $24 \mathrm{~h}$, and free calcium was determined colorimetrically using a spectrophotometer (Shimadzu Corporation, Kyoto, Japan). The protein content of the cultures was measured and corrected for the following extraction using $1 \mathrm{mM} \mathrm{NaOH}$ in $0.1 \%$ sodium dodecyl sulfate.

Experimental grouping. All parameters obtained in the current study were classified as i) normal group; ii) ALD (1.5 mmol/l) group; iii) siRNA transfection (ALD + OSX-siRNA) group, using siRNA-transfected cells that were effectively inhibited by the above screening and optimization methods; and iv) negative transfection control (ALD + negative control siRNA) group, using siRNA-transfected cells that were ineffectively inhibited using the same methods. Following 3-7 h transfection, the medium was replaced by ALD-containing medium in all groups except the normal group. Total RNA was extracted at 24 and $48 \mathrm{~h}$ subsequent to transfection, and total protein was extracted at 48 and $72 \mathrm{~h}$ subsequent to transfection. The transfection rate was determined using FAM fluorescence labeled non-sense siRNA (Thermo Fisher Scientific, Inc., Waltham, MA, USA). The siRNA sequences used were as follows: Sense strand (5-3), UACAAGUACUGGUUGAAC CTT; and anti-sense strand, GGUUCAACCAGUACUUGC ATT. siRNA was synthesized by Shanghai GenePharma Co., Ltd., Shanghai, China).

siRNA transfection. VSMCs were seeded into a 6-well plate and cultured in $0.5 \mathrm{ml}$ serum-free Dulbecco's modified Eagle's medium (DMEM; Gibco; Thermo Fisher Scientific, Inc.) without antibiotics at a density of $75-90 \%$ according to the manufacturer's protocol of Lipofectamine ${ }^{\mathrm{TM}} 2000$ (Thermo Fisher Scientific, Inc.). siRNA and the transfectant in each well were diluted with $50 \mu 1$ serum-free DMEM, mixed gently and incubated at room temperature for $6 \mathrm{~min}$. The diluted siRNA and transfectant were then mixed and incubated at room temperature for $25 \mathrm{~min}$. The $120 \mu \mathrm{l}$ mixture was directly added to each well and mixed gently by vibrating the plate, and then cultured in a $\mathrm{CO}_{2}$ incubator at $37^{\circ} \mathrm{C}$ for $4-8 \mathrm{~h}$. The serum-free DMEM was replaced by the DMEM containing $10 \%$ fetal bovine serum to continue with culture for additional $24 \mathrm{~h}$ at $37^{\circ} \mathrm{C}$. The DNA $(\mu \mathrm{g}) /$ transfectant $(\mu \mathrm{l})$ ratio was set at 1.0:3.0 during the process of transfection, and the working concentration of siRNA was set at $120 \mathrm{nmol} / 1$.

Reverse transcription-quantitative polymerase chain reaction $(R T-q P C R)$. Total RNA was extracted from cells using RNeasy Mini kit (Qiagen $\mathrm{GmbH}$ ) according to the manufacturer's 
instructions. For each sample, total RNA content was assessed by measuring absorbance at $260 \mathrm{~nm}$ and assessing purity using an A260:A280 ratio. RNA was reverse transcribed and PCR was conducted as described previously (15). All genes were analyzed using the SYBR green detection method with the Stratagene Mx3000P qPCR system (GE Healthcare Life Sciences, Chalfont, UK). Each sample was run in triplicate. The reactions were performed as follows: Initial activation step at $95^{\circ} \mathrm{C}$ for $2 \mathrm{~min}$; denaturation at $94^{\circ} \mathrm{C}$ for $10 \mathrm{sec}$, annealing at $50-68^{\circ} \mathrm{C}$ for $1 \mathrm{~min}$ and extension at $72^{\circ} \mathrm{C}$ for $1 \mathrm{~min} / \mathrm{kb}$, for 40 cycles. All gene expression data were normalized against $\beta$-actin and the control values were expressed as one to indicate a precise fold change value for each gene of interest. The primers used were as follows: OSX, forward 5'-GTC CTCTCTGCTTGAGGAA-3' and reverse 5'-CTTGAG AAGGGAGCTGGGTA-3'; integrin-binding sialoprotein (IBSP), forward 5'-ATGGAGACGGCGATAGTTCC-3' and reverse 5'-CTAGCTGTTACACCCGAGAGT-3'; and $\beta$-actin, forward 5'-TCACCCACACTGTGCCCATCTACGA-3' and reverse 5'-GGATGCCACAGGATTCCATACCCA-3'. The primer sequences were synthesized by Shanghai GenePharma Co., Ltd.

Western blotting. Cells were lysed in radioimmunoprecipitation assay buffer (Invitrogen; Thermo Fisher Scientific, Inc.) containing the cOmplete Protease Inhibitor Cocktail according to the manufacturer's instructions (Roche Diagnostics, Basel, Switzerland). Immunoblotting was conducted as described previously (15). Nitrocellulose membranes were probed overnight at $4^{\circ} \mathrm{C}$ with OSX (1:100; cat. no. ab94744; Abcam, Cambridge, UK) and IBSP (1:100; cat. no. ab84787; Abcam) antibodies, washed in Tris-buffered saline with Tween-20, and incubated with anti-rabbit IgG-peroxidase (Abcam) for $1 \mathrm{~h}$ (1:1,000; Abcam). Control membranes were washed in stripping buffer (Pierce Biotechnology, Inc., Rockford, IL, USA) and re-probed for $1 \mathrm{~h}$ for glyceraldehyde 3-phosphate dehydrogenase expression (1:1,000; cat. no. ab8245; Abcam). Subsequent to washing 3 times for 5 min with Tris-buffered saline Tween 20, the membranes were incubated with the rabbit anti-mouse (cat. no. ab97046) or goat anti-rabbit (cat. no. ab136636) horseradish peroxidase conjugated IgG for $1 \mathrm{~h}$ (Abcam). The immune complexes were visualized using an enhanced chemiluminescence reagent (ECL; GE Healthcare Life Sciences).

Alizarin red staining $(A R S)$. For ARS, cells were washed twice with phosphate-buffered saline (PBS) and fixed with formalin at room temperature for $10 \mathrm{~min}$. Formalin was removed and the wells were washed twice with PBS and once with distilled water. The ARS solution was then added and incubated at room temperature for $30 \mathrm{~min}$. Finally, the wells were washed with distilled water until the background staining on the negative wells (wells containing MSCs) was fully clear. Cells were examined under an inverted optical microscope (Nanjing Jiangnan Novel Optics Co., Ltd., Nanjing, China).

Statistical analysis. All values are presented as the mean \pm standard error. Statistical comparisons were conducted by analysis of variance, followed by Fisher's test using SPSS software (version 17; SPSS, Inc. Chicago, IL, USA). P<0.05 was considered to indicate a statistically significant difference.

\section{Results}

Global transcriptome profiling indicates upregulation of OSX in the calcification of VSMCs. To identify novel mediators of vascular calcification, microarray analysis was conducted on VSMCs induced by ALD culture under calcifying conditions. The result of analysis using Bioconductor limma software demonstrated that 867 genes were upregulated and 376 genes were downregulated $(n=4$, greater than 2 -fold change) following normalization, indicating that the bone-formation-associated gene OSX was differentially expressed at the highest level in calcifying VSMCs (Table I).

Furthermore, gene expression analysis by RT-qPCR validated the observation of OSX enhancement. ALD-induced VSMC calcification was significant subsequent to 6-day culture (Fig. 1A). Associated with this increase in VSMC mineralization, OSX mRNA expression was significantly increased at the same time point $(\mathrm{P}<0.01)$ (Fig. 1B). A significant increase in mRNA expression of the osteogenic marker IBSP was observed at 6 days $(\mathrm{P}<0.001)$, indicating that the transition to the osteoblast phenotype was in progress and thereby validating this in vitro model to study VSMC calcification (Fig. 1C).

Determination of the siRNA transfection rate. Subsequent to transfection of VSMCs at the Lipofectamine transfection dose, green fluorescence was observed under the fluorescence microscope, indicating positive cell transfection. Cells were observed under the inverted microscope, and the cell transfection rate was identified to be greater than 70-75\%, which was sufficient for the requirements of the experiments (Fig. 2).

Impact of OSX SiRNA on the expression of VSMC OSX and IBSP $m R N A$. Following 24-h transfection, the expression of both OSX and IBSP mRNA in VSMCs of the ALD group was significantly higher than that of the normal group $(\mathrm{P}<0.01)$, and the expression of OSX mRNA in the siRNA transfection group was significantly lower than that of the ALD and negative transfection control groups $(\mathrm{P}<0.01)$. No significant differences were observed in IBSP mRNA expression between the three groups ( $P>0.05)$. Subsequent to 48 -h transfection, the expression of OSX mRNA in the siRNA transfection group remained significantly lower than that of the ALD and negative transfection control groups $(\mathrm{P}<0.05)$, however was increased compared with the normal group $(\mathrm{P}<0.01)$. The expression of IBSP mRNA in the siRNA transfection group was significantly lower than that of the ALD and negative transfection control groups $(\mathrm{P}<0.05)$, however was higher than that of the normal group $(\mathrm{P}<0.01)$ (Fig. 3).

Impact of OSX siRNA on OSX and IBSP protein expression in VSMCs. Subsequent to 48-h transfection, OSX and IBSP protein expression in VSMCs of ALD group was significantly upregulated as compared with that of the normal group $(\mathrm{P}<0.01)$; the OSX protein expression in the siRNA transfection group was significantly reduced compared with that of the ALD and negative transfection control groups $(\mathrm{P}<0.01)$. No significant 
Table I. Genes exhibiting the greatest differential expression (log fold change) in vascular smooth muscle cells induced by aldosterone for 6 days in calcifying conditions.

\begin{tabular}{llcr}
\hline Gene ID & \multicolumn{1}{c}{ Gene name } & (Log) fold change \\
\hline OSX & Osterix & 4.11 & $1.23 \times 10^{-14}$ \\
IBSP & Integrin binding sialoprotein & 4.05 & $1.25 \times 10^{-14}$ \\
Trib3 & Tribbles homologue 3 & -2.92 & $3.57 \times 10^{-13}$ \\
Sept4 & Septin 4 & 2.24 & $4.65 \times 10^{-13}$ \\
Art4 & ADP-ribosyltransferase 4 & 2.05 & $1.19 \times 10^{-12}$ \\
Tmem204 & Transmembrane protein 204 & 3.44 & $2.15 \times 10^{-12}$ \\
Trib3 & Tribbles homologue 3 & -2.47 & $3.89 \times 10^{-12}$ \\
Gpr116 & G protein-coupled receptor 116 & 2.57 & $1.69 \times 10^{-11}$ \\
\hline
\end{tabular}

A

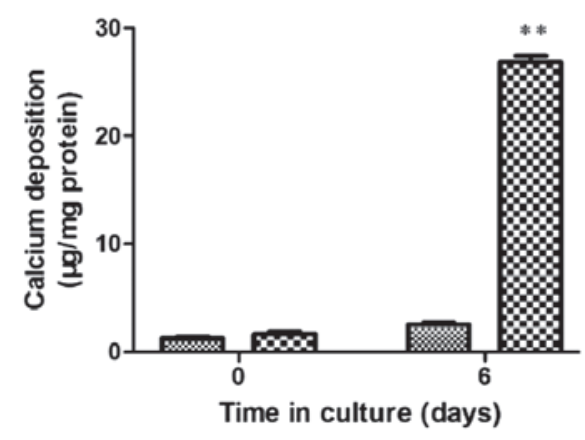

B

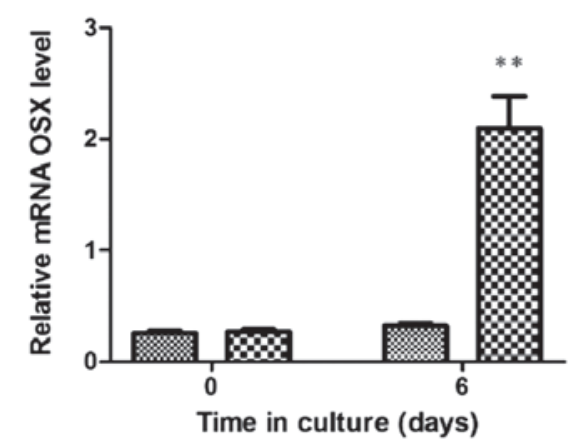

C

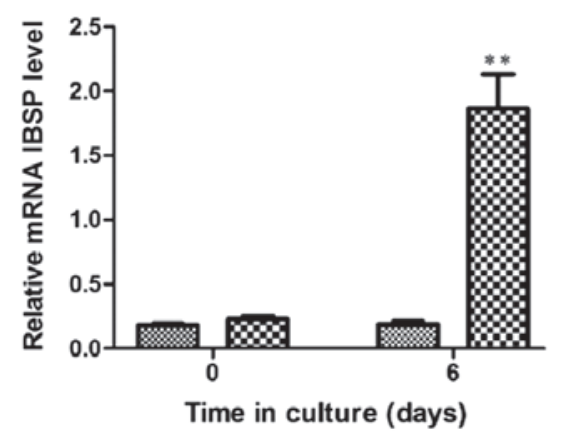

Figure 1. OSX expression during in vitro calcification of murine aortic VSMCs. (A) Calcium content was determined by quantification of $\mathrm{HCl}$ leaching $(\mu \mathrm{g} / \mathrm{mg}$ protein) $(\mathrm{n}=3)$. Fold change in (B) OSX or (C) IBSP mRNA in VSMCs cultured with control or aldosterone $(n=3)$. Results are presented as the mean \pm standard error; ${ }^{* *} \mathrm{P}<0.01$ vs. control. OSX, osterix; VSMCs, vascular smooth muscle cells; IBSP, integrin binding sialoprotein. differences were observed in IBSP protein expression between the three groups $(\mathrm{P}>0.05)$. Subsequent to $72-\mathrm{h}$ transfection, the OSX protein expression in the siRNA transfection group reamained significantly lower than that of the ADL and negative transfection control groups $(\mathrm{P}<0.01)$, however was higher than that of the normal group $(\mathrm{P}<0.01)$. The IBSP protein expression in the siRNA transfection group was significantly lower than that of the ADL and negative transfection control groups $(\mathrm{P}<0.01)$, however was higher than that of the normal group $(\mathrm{P}<0.01)$ (Fig. 4).

Impact of OSX siRNA on calcium salt deposition in VSMCs. No calcium salt deposition was observed in VSMCs at day 6 of transfection in the normal group; large amounts of orange-red calcium salt deposition was observed in the ALD and negative transfection control groups. Calcium salt deposition in the siRNA transfection group was observed to be significantly reduced (Fig. 5).

\section{Discussion}

Stimulation of VSMC phenotype transformation by ALD is widely accepted. ALD can induce VSMCs to express the osteoblast phenotype, i.e. promoting the expression of osteoblast-like genes including OSX and IBSP, and inhibiting the expression of smooth muscle cell-specific genes such as smooth muscle protein 22- $\alpha$, resulting in the induction of calcification of VSMCs. The results of the present study indicated that the expression of OSX and IBSP osteoblast marker proteins was increased markedly with the addition of ALD, indicating that ALD was able to induce differentiation of VSMCs to osteoblasts, which may be one of the important mechanisms underlying vascular calcification in chronic kidney disease.

Despite studies that have improved vascular knowledge, the precise mechanism underlying vascular calcification remains unclear. The current study demonstrated differential expression of novel genes in calcifying murine primary aortic VSMC cultures. Using microarray analysis, functional enrichment analysis and RT-qPCR, it was identified, for the first time to the best of our knowledge, that OSX is upregulated during in vitro VSMC calcification. Mohler et al (16) isolated a group of interstitial cells from the aortic valve stenosed by calcification, and demonstrated that this cell group exhibited similar behaviors to 


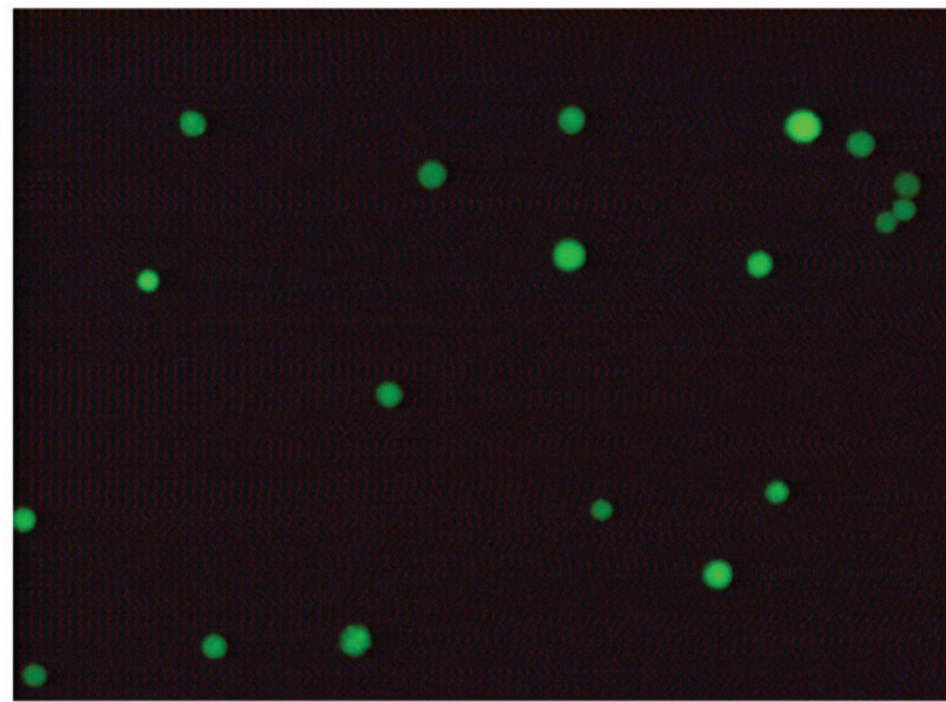

Figure 2. Subsequent to transfection of vascular smooth muscle cells, positive cells were observed as green fluorescence (magnification, $\mathrm{x} 400$ ).

A

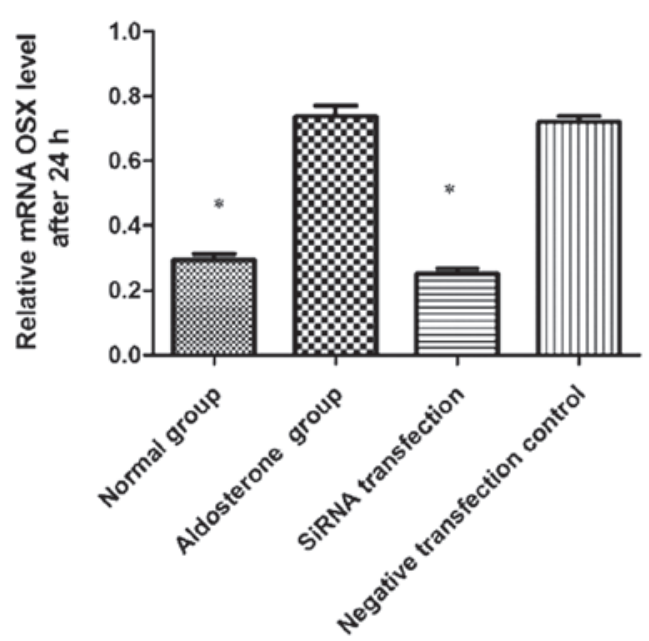

C

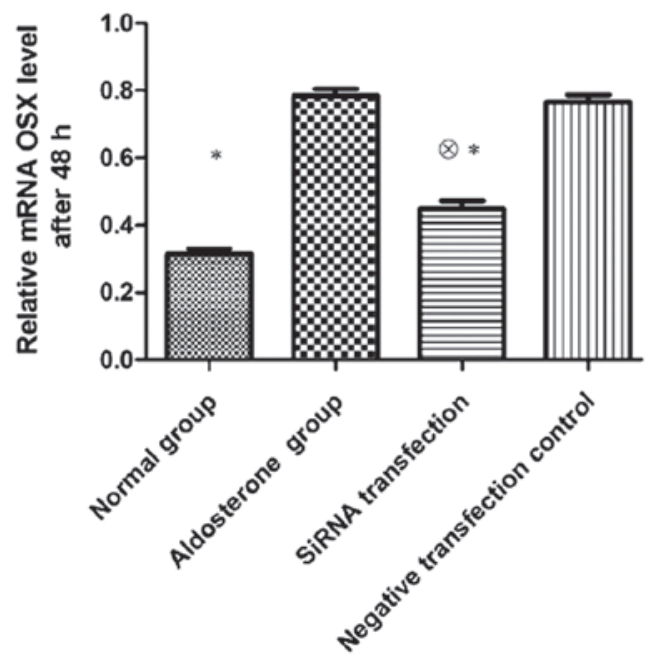

B

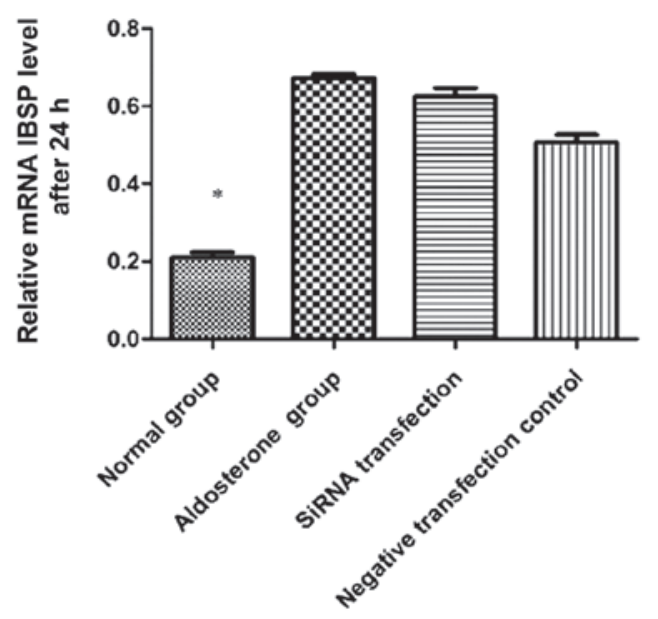

D

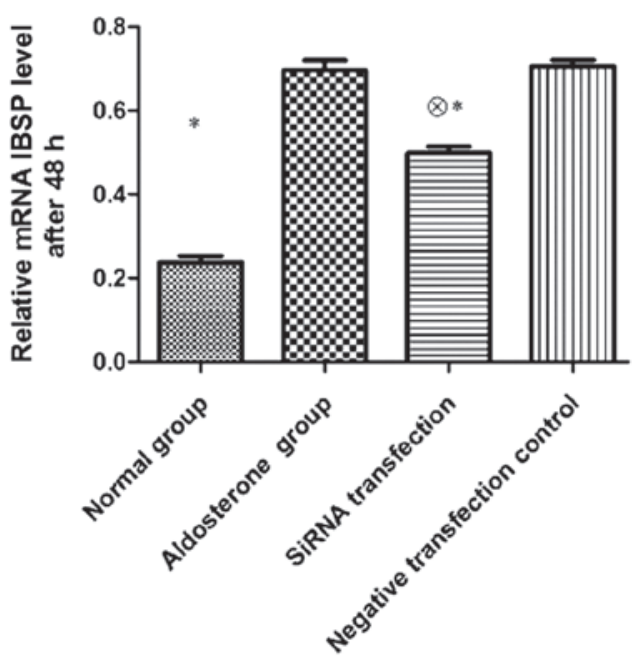

Figure 3. The relative expression levels of OSX and IBSP mRNA in the four groups at 24 and $48 \mathrm{~h}$ subsequent to VSMC transfection during in vitro calcification of murine aortic VSMCs. Fold changes in (A) OSX or (B) IBSP mRNA expression in VSMCs following 24-h transfection ( $\mathrm{n}=3$ ). Fold changes in (C) OSX or (D) IBSP mRNA expression in VSMCs following 48 -h transfection $(\mathrm{n}=3)$. Results are presented as the mean \pm standard error; ${ }^{*} \mathrm{P}<0.01$, vs. aldosterone group at the same time of transfection; ${ }^{\circledR} \mathrm{P}<0.01$, vs. normal group at the same time of transfection. OSX, osterix; IBSP, integrin binding sialoprotein; VSMCs, vascular smooth muscle cells; siRNA, small interfering RNA. 
A

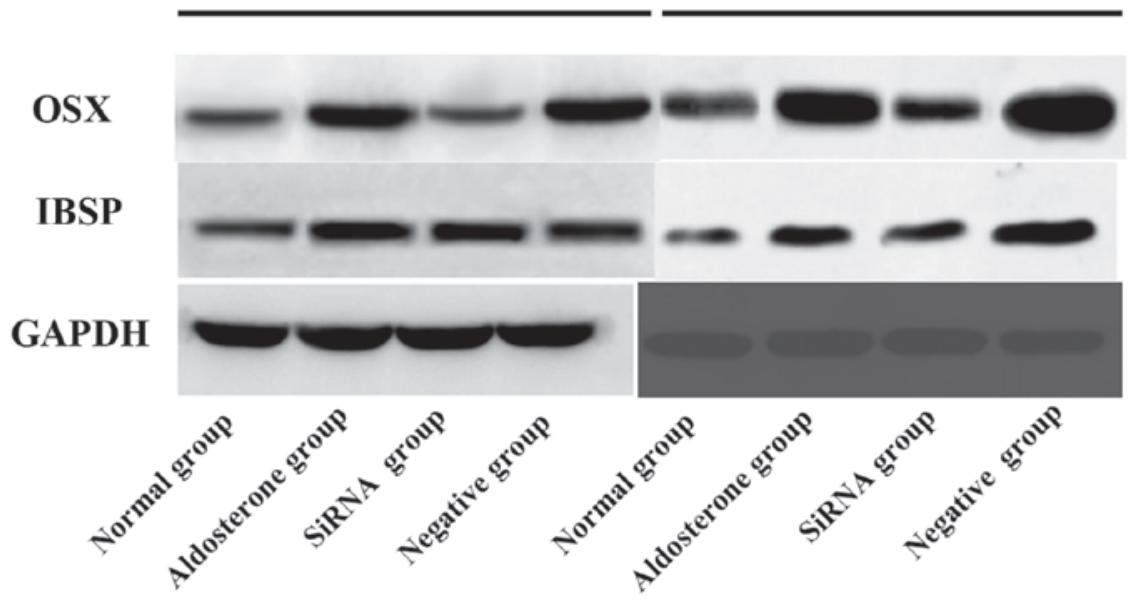

B

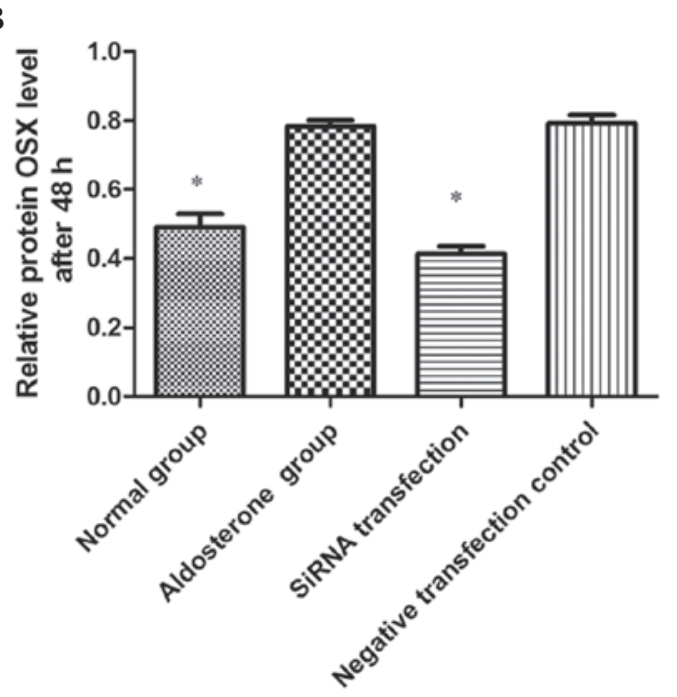

D

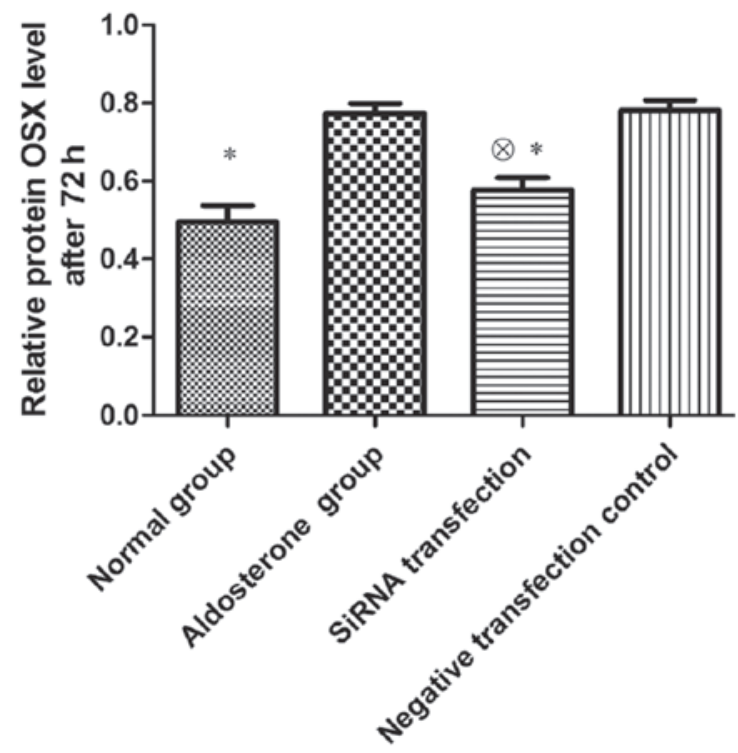

C

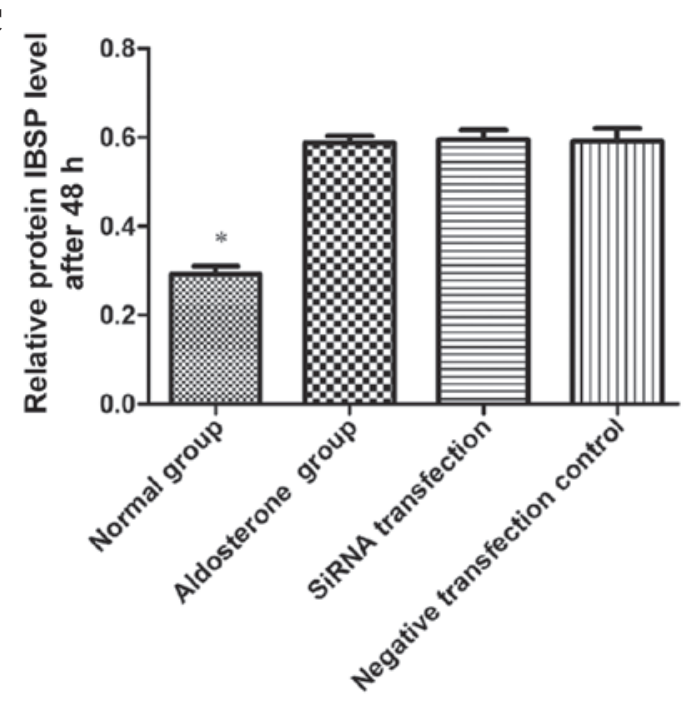

$\mathbf{E}$

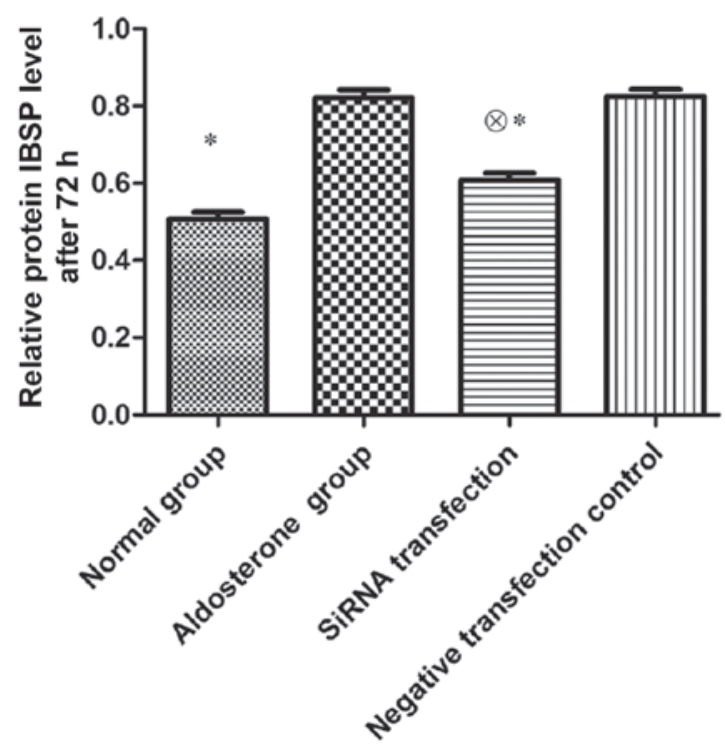

Figure 4. The relative expression protein expression levels of OSX and IBSP protein in the four groups subsequent to VSMC transfection during in vitro calcification of murine aortic VSMCs. (A) Western blot analysis for OSX and IBSP, with GAPDH as the control. Fold changes in (B) OSX or (C) IBSP mRNA expression in VSMCs following 48-h transfection ( $\mathrm{n}=3$ ). Fold changes in (D) OSX or (E) IBSP protein expression in VSMCs following 72-h transfection $(\mathrm{n}=3)$. Results are presented as the mean \pm standard error; " $\mathrm{P}<0.01$, vs. ALD group at the same time of transfection; ${ }^{\otimes} \mathrm{P}<0.01$, vs. normal group at the same time of transfection. OSX, osterix; IBSP, integrin binding sialoprotein; VSMCs, vascular smooth muscle cells; GAPDH, glyceraldehyde 3-phosphate; ALD, aldosterone; siRNA, small interfering RNA. 

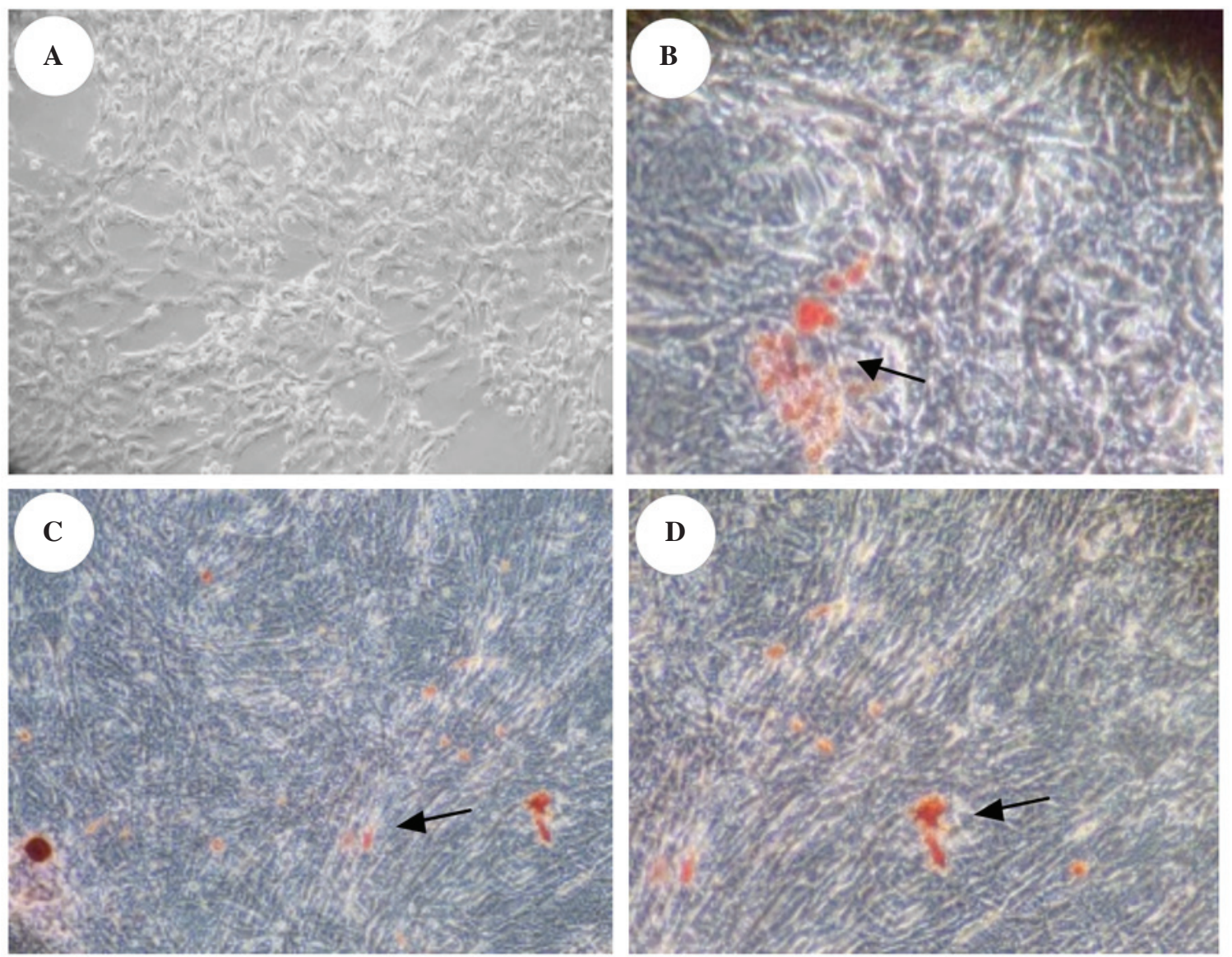

Figure 5. Alizarin red staining of calcium salt deposition in vascular smooth muscle cells of the four groups (magnification, x400). The orange-red color indicates calcium salt deposition. (A) Normal group; (B) aldosterone group; (C) small interfering RNA transfection group; (D) negative transfection control group. The arrows indicate the orange-red calcium salt deposition.

that of vascular smooth muscle cells; they were able to phenotypically transform to osteoblast-like cells and form calcified nodules. Rajamannan et al (17) reported that the expression of multiple osteoblast and bone tissue-associated markers was upregulated in the calcified aortic valves, including OSX, osteocalcin, osteoprotegerin and IBSP. Alexopoulos et al (18) identified that the expression of OSX was increased significantly in the calcified focus of the human aorta. An in vitro study (19) identified that the expression level of OSX protein was elevated significantly following VSMC calcification, and silencing of the OSX gene by siRNA significantly reduced cell calcium deposition and alkaline phosphatase activity. At the same time, VSMC calcification was observed to be blocked. These results suggest that OSX serves an important role in vascular calcification, and its upregulation is associated with the transformation and differentiation of VSMCs to osteoblasts. To further clarify the role of OSX in vascular calcification, the RNAi technique was used to study cultured murine VSMCs, and it was identified that the expression of OSX mRNA and protein in the siRNA transfection group was significantly reduced compared with that of the ALD and negative transfection control groups, indicating that OSX siRNA successfully inhibited the ALD-induced expression of VSMC OSX mRNA and protein in VSMCs at 48 and $72 \mathrm{~h}$.

IBSP is an important non-collagen bone matrix glycoprotein and is important in the development of the osteoblast phenotype. There is an OSX binding site on the promoter of the IBSP-associated bone matrix protein encoding gene. The OSX gene can induce the expression of the IBSP gene, thus promoting cell differentiation to osteoblasts $(20,21)$. It was identified in the current study that when OSX was inhibited by siRNA in a targeted manner, it could downregulate ALD-induced IBSP expression and reduce calcium salt deposition in cells, indicating that OSX serves a role in regulating the downstream associated matrix protein and preventing the occurrence of VSMC calcification.

In the present study, gene chip and gene silencing techniques were used to perform instant transfection using chemically synthesized siRNA, and it was identified that OSX served a key role in the development and progression of ALD-induced VSMC calcification. This observation may aid in the explanation of the role of OSX in the pathogenesis of vascular calcification, however further investigation is required in order to more fully elucidate the mechanism underlying this regulatory effect of OSX.

\section{Acknowledgements}

The current study was financially supported by the Shanghai Commission of Science and Technology (grant no. 12ZR1419000). 


\section{References}

1. Huang L, Teng XY, Cheng YY, Lee KM and Kumta SM: Expression of preosteoblast markers and Cbfa-1 and Osterix gene transcripts in stromal tumour cells of giant cell tumour of bone. Bone 34: 393-401, 2004.

2. Chen MLY and Miao LY: OSX expression during the development of human teeth. Oral Med Res 22: 372-337, 2006.

3. Cao Y, Zhou Z, de Crombrugghe B, Nakashima K, Guan H, Duan X, Jia SF and Kleinerman ES: Osterix, a transcription factor for osteoblast differentiation, mediates antitumor activity in murine osteosarcoma. Cancer Res 65: 1124-1128, 2005.

4. Kim YJ, Kim HN, Park EK, Lee BH, Ryoo HM, Kim SY, Kim IS, Stein JL, Lian JB, Stein GS, et al: The bone-related $\mathrm{Zn}$ finger transcription factor Osterix promotes proliferation of mesenchymal cells. Gene 366: 145-151, 2006.

5. Zhou YS, Liu YS and Tan JG: Is 1, 25-dihydroxyvitamin D3 an ideal substitute for dexamethasone for inducing osteogenic differentiation of human adipose tissue-derived stromal cells in vitro? Chin Med J (Engl) 119: 1278-1286, 2006.

6. Nakashima K, Zhou X, Kunkel G, Zhang Z, Deng JM, Behringer RR and de Crombrugghe $B$ : The novel zinc finger-containing transcription factor osterix is required for osteoblast differentiation and bone formation. Cell 108: 17-29, 2002.

7. Demer LL and Tintut Y: Vascular calcification: Pathobiology of a multifaceted disease. Circulation 117: 2938-2948, 2008.

8. Shioi A: Molecular mechanisms of vascular calcification. Clin Calcium 20: 1611-1619, 2010 (In Japanese).

9. Wu SY, Yu YR, Cai Y, Jia LX, Wang X, Xiao CS, Tang CS and Qi YF: Endogenous aldosterone is involved in vascular calcification in rat. Exp Biol Med (Maywood) 237: 31-37, 2012.

10. Radtke CL, Nino-Fong R, Rodriguez-Lecompte JC, Esparza Gonzalez BP, Stryhn H and McDuffee LA: Osteogenic potential of sorted equine mesenchymal stem cell subpopulations. Can J Vet Res 79: 101-108, 2015.

11. Valenzuela CD, Allori AC, Reformat DD, Sailon AM, Allen RJ Jr, Davidson EH, Alikhani M, Bromage TG, Ricci JL and Warren SM: Characterization of adipose-derived mesenchymal stem cell combinations for vascularized bone engineering. Tissue Eng Part A 19: 1373-1385, 2013.
12. Staines KA, Zhu D, Farquharson $C$ and MacRae VE: Identification of novel regulators of osteoblast matrix mineralization by time series transcriptional profiling. J Bone Miner Metab 32: 240-251, 2014

13. Irizarry RA, Bolstad BM, Collin F, Cope LM, Hobbs B and Speed TP: Summaries of Affymetrix GeneChip probe level data. Nucleic Acids Res 31: e15, 2003.

14. Kauffmann A, Gentleman R and Huber W: ArrayQualityMetrics-a bioconductor package for quality assessment of microarray data. Bioinformatics 25: 415-416, 2009.

15. Macrae VE, Horvat S, Pells SC, Dale H, Collinson RS, Pitsillides AA, Ahmed SF and Farquharson C: Increased bone mass, altered trabecular architecture and modified growth plate organization in the growing skeleton of SOCS2 deficient mice. J Cell Physiol 218: 276-284, 2009.

16. Mohler ER III, Wang H, Medenilla E and Scott C: Effect of statin treatment on aortic valve and coronary artery calcification. J Heart Valve Dis 16: 378-386, 2007.

17. Rajamannan NM, Subramaniam M, Rickard D, Stock SR, Donovan J, Springett M, Orszulak T, Fullerton DA, Tajik AJ, Bonow RO and Spelsberg T: Human aortic valve calcification is associated with an osteoblast phenotype. Circulation 107: 2181-2184, 2003

18. Alexopoulos A, Bravou V, Peroukides S, Kaklamanis L, Varakis J, Alexopoulos D and Papadaki H: Bone regulatory factors NFATc1 and Osterix in human calcific aortic valves. Int J Cardiol 139: 142-149, 2010.

19. Taylor J, Butcher M, Zeadin M, Politano A and Shaughnessy SG: Oxidized low-density lipoprotein promotes osteoblast differentiation in primary cultures of vascular smooth muscle cells by up-regulating Osterix expression in an Msx2-dependent manner. J Cell Biochem 112: 581-588, 2011.

20. Samee N, Geoffroy V, Marty C, Schiltz C, Vieux-Rochas M, Levi G and de Vernejoul MC: Dlx5, a positive regulator of osteoblastogenesis, is essential for osteoblast-osteoclast coupling. Am J Pathol 173: 773-780, 2008.

21. Igarashi M, Kamiya N, Hasegawa M, Kasuya T, Takahashi T and Takagi M: Inductive effects of dexamethasone on the gene expression of Cbfa1, Osterix and bone matrix proteins during differentiation of cultured primary rat osteoblasts. J Mol Histol 35: 3-10, 2004. 